\title{
Appendix 2: The Seventy Principles of the Universal Constitution
}

\author{
L'homme et le contrat social
}

I.

L'homme reçoit de la société le seul développement de sa faculté appétitive, matérielle et intellectuelle qui est: L'instinct mûri et formé par la société.

II.

Pour que cette recette de la société soit une recette réelle et obligatoire, il faut qu'elle ait la liberté d'employer tous ses efforts pour satisfaire ses desirs de la manière. la plus complette, ou pour atteindre son suprême bonheur, conformément au développement de sa faculté appétitive.

III.

Cette liberté de chaque individu est l'égalité des droits la plus parfaite.

IV.

Personne ne peut aliéner cette égalité libre ou liberté égale, sans se déclarer prodigue ou foible, trompé ou forcé, cas dans lesquels tout contrat d'aliénation est nul de fait, parce que la faculté appétitive cessant par cette aliénation d'être un avantage réel, cesseroit aussi d'être obligatoire.

V.

Il est clair que le développement de la faculté appétitive intellectuelle doit suivre les mêmes principes. Toute formule coercitive de penser ou de croire est donc une infraction à la liberté égale et à l'égalité libre.

VI.

Dans le seul cas où les efforts d'un individu empêcheroient les efforts de l'autre, l'usurpation de la liberté égale doit être réprimée par la loi, afin de mettre en sûreté les droits que tous les individus de la société ont au bonheur.

VII.

Les loix ne sont donc et ne doivent être que la garantie de la liberté et de l'égalité, ou du droit de l'exercice de tous ses efforts, pour atteindre le suprême bonheur dans la société.

VIII.

Sans ce droit, le développement de la faculté appétitive cesse d'être un bénéfice réel: et dans ce cas-là l'individu ne doit plus rien à la société ; et la loi devenue trop foible pour garantir ses droits, met hors de responsabilité sa rébellion qui n'est alors que la sainte résistance à l'oppression. Oui, elle sanctifie son insurrection, et lui en fait un devoir. Ses facultées développées et puis réprimées par la force ou la ruse justifient sa vengeance, et demandent la punition des tyrans par ces mêmes principes qui sanctifient les loix et les peines. 


\section{Principe, Droits \& Égoïsme}

I.

La conservation individuelle, est le principe de tout droit, et de tout ce qui est contre droit.

II.

Dans l'état de nature le devoir de la relation, n'a lieu qu'en vers les membres $d u$ moi individuel. Dans l'état de société, au contraire, il se porte sur les mêmes membres du moi social avec la même latitude que dans l'état de nature, où il agit sur tous les membres du moi individuel. Et comme, dans l'état de société, l'homme a besoin de la relation de ses semblables pour la conservation individuelle, et la satisfaction de la faculté appétitive développée, le besoin établit le devoir de la relation de son côte, et fait un droit de cette réciprocité nécessaire.

III.

Le droit naturel, est le devoir pour le principe.

IV.

Le droit civil et politique, est le devoir de la relation pour la conservation individuelle, qui est, et qui reste aussi son principe éternel.

V.

Toute contribution nécessaire à la conservation individuelle, que la majorité exige de la minorité en vertu du principe, est fondée en droit; étant le devoir de la relation pour le principe.

VI.

L'égoïsme est aussi la conservation individuelle, mais qui refusant la réciprocité de la relation, refuse son devoir, n'est pas en droit. La conservation individuelle est donc le principe éternel, la bâse fondamentale de toute justice et injustice (i).

VII.

Toute société, dont la majorité commande à la minorité un devoir, un sacrifice du moi individuel, sans que le moi social en exige la nécessité, s'éloigne du droit en s'éloignant du devoir de la relation pour le principe. Elle tombe dans le refus de la réciprocité de la relation ; dans l'égoïsme, principe infâme, étant aussi la conservation individuelle; mais dont la ligne de démarcation trop déliée, n'est distinguible qu'aux connoisseurs, se glisse bientôt dans la société comme véritable principe, dont il détruit la divinité d'un intérêt commun; et en introduisant l'enfer d'un intérêt partiel, engloutit le droit véritable; et en vomissant l'exécrable droit du plus fort dans la société, rend l'un coupable, et l'autre misérable, confond l'erreur avec la vérité, obscurcit le sentier simple de la nature et de la raison, en compliquant la philosophie sociale, et en trompant sur-tout l'humanité dans la science de vivre.

\section{Moralité de l'individu}

I.

La moralité est la relation volontaire partielle pour le principe (I) d'un autre.

(I) Toutes les fois que nous parlerons dans la suite du principe en général, nous entendrons la conservation individuelle, définition du principe général de tout être créé, comme nous l'avons posé dans le troisième chapitre. 
II.

Aucune moralité n'est possible envers la société; chaque sacrifice n'agit qu'en droit, parce qu'il se porte toujours réciproquement sur le moi individuel.

III.

Dans l'état de nature, la moralité n'est autre chose qu'une liberté poussée trop loin, une licence, un crime. L'étincelle morale court en lui, pour ainsi parler, mais l'exercice de la relation volontaire (la moralité) dans l'état de nature ne peut le conduire qu'à sa perte. L'heureux lien de la société ne l'a pas encore attaché à son prochain, ne le connoissant pas encore, il ne peut avoir aucune relation volontaire avec lui, et l'exercice de son impulsion morale est encore contre le droit.

IV.

La moralité qui est une licence, un crime pour l'homme dans l'état de nature, devient sa plus haute vertu dans l'ordre social, et le témoignage le plus auguste, le plus irrécusable, qu'il est né pour la société.

V.

L'égoïsme est l'opposé de la morale, celui-ci accepte sans vouloir rendre, celle-là donne sans vouloir qu'on lui rende.

\section{Moralité et immoralité de la société}

I.

La moralité d'état est une chose partielle qui n'a qu'une existence isolée, bornée dans le partiel. Elle est la plus haute vertu de l'humanité qu'elle honore; mais liée aux principes conservateurs du grand tout, empiétant sur le grand moi de la société, elle affoiblit, elle énerve, elle tue le droit, elle est un crime que les loix doivent réprimer et punir (I).

(I) La loi contre les émigrés n'est pas un acte immoral, mais au contraire bien fondé en droit.

\section{Du droit du plus fort et du droit de la ruse}

I.

Le droit du plus fort et le droit de ruse sont des droits sacrés, comme défense pour le principe.

II.

Le droit du plus fort est une tyrannie; le droit de la ruse est un assassinat, comme offense contre le principe.

III.

Cette différence constitue les deux sortes de majorité: l'une comme défense pour le principe, agit en véritable droit, et peut même sacrifier la minorité, étant un moyen conservateur pour la partie plus grande du corps du moi social; et l'autre comme offense contre le principe, agit en droit du plus fort, qui n'est qu'une tyrannie, et qui transmet le véritable droit à la minorité, qui peut se servir alors de tous les mêmes moyens, pour abattre la majorité rébelle, agissant toujours en véritable droit. 


\section{Loi, Pouvoir, Corps législatif}

I.

La loi est l'expression de la détermination des facultés morales, dirigées par le principe, et pour le principe.

II.

Le pouvoir n'est qu'un seul. Il est législatif et exécutif en même-temps, car il est la mesure déterminative et le régulateur du corps législatif, des facultés morales.

III.

Le corps législatif est le délégué du peuple, pour représenter les facultés morales, le sentiment et la raison, pour chercher et pour exprimer la détermination par le principe et pour le principe.

IV.

Le corps législatif étant obligé de déterminer par le principe et pour le principe, il est donc aussi chargé de l'exercice du seul pouvoir, il est donc en même-temps comme le pouvoir luimême, législatif et exécutif.

V.

Si-tôt que le corps législatif cesse de représenter ces facultés morales en s'écartent de leur marche naturelle, il cesse d'être corps législatif entièrement comme particulièrement. Sa mission est finie, puisque son but est manqué. Il est rappellés par le pouvoir (I). (I) Au peuple seul appartient la sanction des loix; mais comme chaque loi doit être précédée d'un considérant explicatif des principes du droit, sur lequel cette loi se fonde ; que le peuple n'en peut rejetter ni recevoir aucune, qu'en faisant précéder également sa décision d'un semblable considérant; pour mettre un frein aux entreprises du corps législatif, il suffira de poser en principe, $\mathrm{i}^{\circ}$, que chaque loi, comme chez les Romains, aura force jusques au rassemblement des Sections populaires, qui aura. lieu de trois mois en trois mois. $2^{\circ}$. Que chaque loi sera rendue par appel nominal, lequel sera envoyé avec la loi aux sections populaires, où il sera proclamé. $3^{\circ}$. Que lorsqu'un délégué aura voté pour trois loix, que le peuple conduit par le considérant explicatif des principes du droit, posé dans la première partie de la Constitution aura rejetées, le Secrétaire de la Section populaire fera passer au corps législatif le procès-verbal, qui établit que tel délégué a voté trois fois contrairement au principe, et sans autre formalité, le membre sera exclu de l'assemblée législative. Ce moyen aussi simple que juste, ne sera sujet â aucun inconvénient, puisque le membre exclu, sera exclu par le principe, seul pouvoir légitime.

VI.

Si-tôt que le corps législatif cesse de se faire déterminer, par cette autorité médiatice, par le principe, ses résultats cessent d'être loi.

\section{Du souverain}

I.

Dans l'état de nature, l'homme individuel étant indépendant hors de soi, est un tout parfait, un être souverain.

II.

Dans l'état de culture, l'homme individuel étant devenu dépendans des autres, cesse d'être un tout parfait. Sa souveraineté est passée à la société entière. 
III.

La propriété du souverain primitif de l'homme, dans l'état de nature, consiste dans toutes les forces et moyens naturels qui peuvent conduire l'homme à son but, exigé par le principe.

IV.

La propriété du nouveau souverain de la société, consiste dans toutes les forces et moyens de culture, qui se réunissent aux forces et moyens naturels antérieurs de chacun de ses membres.

V.

La contribution, est l'emploi de toutes les forces et moyens naturels (de toute sa propriété dans cet état) pour atteindre le but exigé par le principe, lesquels constituent les devoirs et les fonctions du souverain primitif, dans l'état primitif.

VI.

Ces devoirs et obligations du souverain primitif, peuvent être requis tant qu'ils restent moyens pour le but, et qu'ils ne sont pas contre le but, car ils agiraient alors contre le principe; et comme le principe est le seul pouvoir, seul, il peut exiger une contribution, un devoir. C'est donc hors du pouvoir en droit du souverain légitime, de commettre une action quelconque contre le principe et par conséquent, il n'est nullement du droit du souverain de se faire mal à lui-même, quoiqu'il puisse le faire.

VII.

Dans l'état de culture, les droits du nouveau souverain s'étendent sur ses membres dans la même mesure. Toutes les contributions et devoirs qu'il exige, ne doivent être que pour le but, et non lui être contraires, pour le principe, qui demeure le seul pouvoir, et par conséquent le pouvoir de faire mal à lui-même est hors du droit du souverain quoiqu'il le pût faire. Cette distinction entre pouvoir faire en droit ou contre droit, pouvoir agir contre le principe ou pour le principe, constitue seule la différence absolue entre la liberté et la licence.

VIII.

La conduite du souverain primitif dans cette contribution de ses forces et moyens naturels pour le principe, est une offre entière sans nulle économie ou restriction, il suit constamment la loi inviolable qui dit: Que cela soit: la victoire ou la mort.

IX.

La conduite du souverain primitif, dans l'état de nature, est la sainte et inviolable formule du nouveau souverain de la société. Dans l'état de culture, quand il s'agit d'une contribution de tous les moyens de culture (des propriétés sociales) pour défendre son but, exigé par le principe, nulle économie, nulle restriction, il faut employer le tout pour défendre le tout.

\section{$\mathrm{X}$.}

Le nouveau souverain occupant la place du souverain primitif, et entrant dans tous ses droits, aucuns de ses membres individuellement, nulle corporation, quelle qu'elle soit, ne peut avoir aucun prétexte pour s'approprier un intérêt inégal ou plus important, quand même on voudroit ou pourrait faire davantage pour cet intérêt plus important, puisqu'on raison de l'acquisition de sa faculté appétitive, tant matérielle qu'intellectuelle, perfectionnée et développée, chaque membre, dans cet état est redevable au nouveau souverain de tout ce dont il peut concourir par ces nouveaux moyens de culture, ainsi que guidé par son instinct animal, il l'aurait fait autrefois pour son souverain primitif, l'être-homme dans l'état de nature. 
XI.

Quoiqu'il soit bien avéré qu'aucun individu isolé en son particulier, ni toute autre corporation sous quelque unité nouvelle adoptée qu'elle subsiste, ne puisse pas acquérir dans la société un intérêt inégal et plus important. Il n'est pas moins constant que ce nouveau souverain luimême, n’a pas le droit de céder ou transporter à un individu isolé ce nouvel intérêt inégal et plus important, quelque soit cet individu, ni même à une corporation quelconque, sous quelque dénomination qu'elle existe, c'est-à-dire que le nouveau souverain ne peut abuser du trésor social.

\section{XII.}

L'établissement ou l'admission d'un second intérêt; privilège différent pour un individu où corporation, étant le renversement de la bâse naturelle de l'unité d'intérêt, tant de l'ancien que du nouveau souverain, puisque ce nouvel intérêt ne peut se nourrir qu'au détriment de l'autre, il en résulte que cette introduction d'un second intérêt; sort du cercle du juste pouvoir de l'ancien et du nouveau souverain, et qu'elle dégénère en usurpation et tyrannie, ainsi seroit le suicide, auquel l'ancien et le nouveau souverain pourroit également se livrer mais dont aucun n'osera faire usage en droit.

XIII.

L'établissement en introduction d'un intérêt second et différent, privilège pour un individu isolé ou une corporation quelconque un roi ou sénat héréditaire, étant une aliénation et abus du trésor social, est nécessairement par sa propre nature, une lésion du droit de propriété des autres membres du grand tout, et ensemble parfait du nouveau souverain, parce que cette aliénation et abus du trésor social, ne sont pas plus autorisés par l'existence du nouveau souverain, que le souverain primitif poussé par son instinct animal, n'auroit été autorisé à l'entreprendre contre un, ou plusieurs de ses membres.

\section{Contributions publiques}

I.

Dans le souverain primitif, aucun de ses membres ne sollicite ni n’usurpe la délégation ou les fonctions des autres, puisqu'au lieu d'y gagner, il se chargeroit uniquement d'une peine plus grande. Ainsi c'est l'instinct, la nécessité qui règle le régime dans le souverain primitif, et qui oblige alternativement l'un de commander, et l'autre d'obéir.

II.

Il n'en est pas ainsi pour le nouveau souverain (la société); car ici l'intérêt qu'ils y pourraient trouver fait craindre à chaque instant l'usurpation d'un ou de plusieurs membres réunis, ou du moins la rend très possible. Il faut que la justice, les connoissances de l'instinct mûri et perfectionné exercent par rapport au grand tout, la surveillance que nous voyons l'instinct animal observer dans l'état de pure nature, c'est-à-dire, que par la fixation du maximum contributionnel des moyens de chaque membre représentant la nécessité générale du Corps entier, ils mettent un obstacle, ou déjouent toutes les entreprises de ces agens représentatifs, contre la sûreté, ou l'existence du grand tout.

III.

Quand l'être homme, ce tout singulier et particulier, s'est réuni à plusieurs êtres de sa nature pour former son instinct et le leur, et par là multiplier mutuellement leur jouissances, il a voulu gagner; mais il n’a prétendu rien perdre, ni renoncer à rien par cette réunion. Cela seroit contre le principe. 
IV.

Le nouveau souverain (la société) ne peut donc jamais exiger de lui comme une contribution nécessaire soit morale ou physique, et palpable même, la moindre obéissance, action, sacrifice, fût-il de son abondance ou luxe moral ou physique, si le sacrifice n'est pas évidemment et indispensablement nécessaire dans le moment, pour la défense du principe du moi social, du nouveau souverain, auquel la souveraineté primitive a été transmise et dont il est devenu un membre dépendant. L'obéissance envers chaque fonctionnaire représentant au moment de sa fonction pour le souverain et pour le principe; chaque sacrifice exigé de sa propriété pour la nécessité évidente et indispensable du soutien et de la défense du souverain pour le principe est fondé en droit, et doit être rendu sans restriction; mais dans le cas contraire, ou dans toute autre occasion, la moindre obéissance ou préférence qu'un membre exigera involontairement d'un autre, ou même le moindre sacrifice contributionnel du besoin ou du luxe, que le gouverne' ment exigera d'un individu, seroit une tyrannie, une licence, un attentat à la propriété individuelle. En outre, comme il n’a jamais renoncé à sa souveraineté primitive, que la nature ne s'est rien moins qu'engagée à payer aux dépens de sa propriété et de ses propres fonds les dettes contractées par l'art, qu'il est bien plus juste, au contraire, que la nature surveille toujours cet être nouveau l'art, afin d'empêcher la dilapidation de ses facultés; il en résulte qu'on ne peut jamais ordonner à un des membres de l'association, la déposition des armes qui servent à sa défense personnelle, ni l'en priver. Ainsi dans le cas d'usurpation des biens individuels du souverain primitif, on ne peut sous aucun prétexte exiger d'un individu le sacrifice de ses moyens de défense, ni le faire renoncer à la faculté d'en user. A moins que la défense du nouveau souverain (la société) ne soit tellement confondue dans la défense du souverain primitif l'être-homme, c'est-à-dire, qu'il s'agisse pour le principe de la conservation de la base naturelle, la liberté et l'unité d'intérêt. Parce qu'alors la querelle intéresse à la fois le corps entier, que tous combattent pour le tout, et que le tout doit intéresser tous; ou le moi individuel est changé et remplacé par le moi social.

V.

Dans cette hypotèse, le souverain primitif, (l'être-homme) sert encore de modèle au nouveau souverain la société, parce que touchant la faculté d'éloigner le mal et les efforts pour parvenir au souverain bien, le triomphe de l'un est aussi le triomphe de l'autre, et que la perte du premier, entraîne après elle la chute du second. Alors l'abandon de la faculté de se défendre soi-même, l'enlèvement des armes, et le sacrifice de tous moyens de défense personnelle, qui ne peuvent en aucuns cas être exigés d'un membre de l'association comme contribution, peuvent lui être imposés comme peines, et ce cas arrive lorsque la liberté d'un individu ayant dégénéré en coupable licence, elle entrave l'exercice de la juste liberté d'autrui, ce qui peut se rencontrer surtout dans l'état social, ou un membre pouvant usurper l'intérêt égal des autres, il finiroit par rendre dépendant le souverain primitif (l'être-homme), lequel n'a jamais pré, tendu résigner sa souveraineté individuelle, 
VI.

Quand aux membres fonctionnaires représentans, il nous faudra bien exactement suivre A leur égard le procédé du souverain primitif, et notamment dans le cas où ils tiennent toutes les autres forces et ressources comme enchaînées, et ce, dans l'attente de la fin de l'opération, que la nécessité de sauver le corps entier peut leur prescrire quelquefois dans des circonstances délicates. Il faut que malgré cette résignation apparente, tous les autres membres tiennent préparés tous les moyens de défense dont ils ne doivent jamais se dépouiller, et qu'ils soient dans un tel état d'application et d'examen intérieur sur le membre qui agit, qu'ils ayent plutôt l'air d'attendre la fin de l'opération avec calme et dignité, que de la servir; et l'on doit en agir avec d'autant plus de rigueur sur ce chapitre dans l'état social, qu'on a à redouter ici tous les accidens qui sont à craindre pour le souverain primitif, dans une situation pareille.

\section{Gouvernement}

I.

Dans l'état primitif de pure nature, tons les membres concourent au besoin et au contentement de tout le corps, aucun ne s'en dispense par privilège, aucun ne s'y porte par préférence, et nul ne peut demeurer dans une coupable neutralité, quand il s'agit de leur égal intérêt relativement au but, au principe.

II.

Tous les membres de la société doivent concourir de tous leurs moyens au besoin et au contentement du tout. Aucun ne peut avoir le privilège de s'en dispenser: aucun ne doit s'y immiscer par préférence, et nul ne peut rester dans une inertie ou neutralité coupable, quand il s'agit de l'intérêt égal relativement au but (le principe).

III.

Le gouvernement de la société doit être d'après le régime de la nature, qui est l'unique forme de gouvernement juste et exacte.

IV.

Chaque fonctionnaire public est un véritable représentant du peuple, dont l'élection se fait par le peuple même, ou par un corps électoral, élu par le consentement général, et muni de pouvoirs pour l'élection des fonctionnaires.

V.

L'élection de chaque membre du corps législatif inclusivement, tous les fonctionnaires, si petit que soit leur emploi, se doit faire par appel nominal nominal à haute voix, et aucun fonctionnaire ne pourra parvenir à aucune place sans cette formalité.

VI.

Le terme des représentations principales et en chef, doit être le plus court possible, et une prolongation occasionnée par une réélection illimitée n’y a pas lieu.

VII.

La séparation, et le morcellement des pouvoirs, et des forces nécessaires dans les agens représentatifs principaux et en chef est inévitable, et de toute nécessité dans le corps social. 
VIII.

Tous les représentans du peuple en chef, doivent être non-seulement de la durée la plus courte possible, mais il faut aussi que la surveillance générale du peuple porte toute son attention sur ses fonctionnaires en chefs, qui durant le court espace de leurs fonctions, semblent réunir tous les moyens de la société. II faut que le peuple entier soit dans un tel état de réunion et de vigilance, et garde un calme tel et majestueux, qu'il semble plutôt les surveiller, que les servir en esclave. C'est ainsi que le peuple reste toujours en même tems le monarque et le sujet.

IX.

Les représentans subalternes de la force $M$ se suppléent dans la nature l'un à l'autre. En cas de besoin, le membre le plus proche agit pour l'autre. Lorsqu'il se passe dans un endroit du territoire de la société un événement décisif pour le corps entier, où il est besoin d'une représentation rapide et instantanée de la force d'un membre absent, ou que par impossibilité, le peuple entier ne peut pas être présent lui-même, alors le district et département, commune, section, individu, enfin le membre le plus à portée doit, sans contredit, suppléer les autres en droit strict et véritable, parce qu'il est en devoir pour le principe.

$\mathrm{X}$.

Les seules fonctions que le peuple entier, le souverain est en état d'exercer lui-même, sont la défense pour son moi social, les élections de ses représentans, la confiance et la tranquillité générale, l'insurrection et la surveillance générale.

\section{Liberté}

I.

Liberté est, l'égalité des droits.

II.

Chaque liberté, par préférence d'un membre ou corporation quelconque, est une puissance partielle aux dépens d'autrui, au détriment du principe, est licence.

III.

Tout ce qui est contraire au principe est une liberté liberticide; une licence, et licence que le souverain n'a pas le pouvoir de souffrir, soit qu'une telle licence se cache sous le masque de la liberté d'opinions, de la presse, de la politique, de la religion, qu'elle se nomme tolérance, ou sous quelque forme qu'elle se présente; elle est attentatoire au corps social dans son intégrité, et doit être punie de mort.

\section{Egalité}

I.

L'égalité des droits, est la véritable liberté l'un chacun.

II.

Chaque législateur, voulant forger une égalité, de manière à satisfaire l'inégalité des nuances de la faculté appétitive, découlant de la différence naturelle des diverses organisations, surpasse les bornes de son pouvoir, agir contre 
droit, et improuvé par la nature, repoussé par le principe, même sous le prétexte d'améliorer par des loix les mœurs, la moralité des individus; parce que chaque action forcée cesse d'être moralité, en cessant d'être un acte volontaire: et devient de la part du législateur, tyrannie, n'ayant pas le droit d'enchaîner le droit égal de la plus parfaite liberté d'un chacun de satisfaire sa faculté appétitive d'une manière différente, selon son organisation et son plaisir licite.

III.

Chaque loi qui exige des sacrifices involontaires, concernant le luxe, l'abondance, (excepté dans les momens impérieux, où ils sont exigés par la nécessité pour le salut public), est attentatoire à la propriété individuelle, est oppressive, vexatoire, criminelle, est repoussée par le principe.

IV.

Le partage des terres ne pouvant jamais se trouver dans le cas d'être exigé pour le soutien et la défense du principe, il reste toujours hors du pouvoir du souverain, et ne peut avoir lieu de nulle manière dans une législation.

\section{Éducation}

I.

Le principe d'une république démocratique, est, la vraie connoissance des charmes et avantages réels qu'elle nous donne.

II.

Pour élever la jeunesse en républicains démocrates, il n’y a d'autre méthode, que d'avoir une constitution instructive et convaincante, qui conduise la jeunesse républicaine au but nécessaire, d'après la mesure de la nature, par les attraits du plaisir.

\section{Peines et délits}

I.

Les peines sont fondées dans la nature, et doivent être en proportion avec les délits; elles doivent être fixées par degrés.

II.

La Société ne peut punir aucun de ses membres de la peine de mort, s'il n'a commis un délit contre le corps entier. Le moi individuel étant dans l'état de culture changé dans le moi social; et le principe, la conservation, individuelle, étant devenu la conservation individuelle de la société entière, il en résulte, que l'on ne peut commettre un crime contre le principe, si l'on ne le commet point contre le corps de la société entière. 